\title{
Adesão ao autocuidado de pessoas com Diabetes Mellitus na Atenção Primária: estudo de método misto
}

\author{
Adherence to self-care in people with Diabetes Mellitus in Primary Care: a mixed-methods study \\ Adhesión al autocuidado de personas con Diabetes Mellitus en la Atención Primaria: un estudio de \\ método mixto
}

Samara Eliane Rabelo Suplici ${ }^{1}$ Betina Hörner Schlindwein Meirelles ${ }^{1}$ (1) Denise Maria Guerreiro Vieira da Silva ${ }^{1}$ (D) Julia Estela Willrich Boell ${ }^{1}$ (D)

1. Universidade Federal de Santa Catarina, Centro de Ciências da Saúde, Departamento de Enfermagem. Florianópolis, SC, Brasil.
Autor correspondente: Samara Eliane Rabelo Suplici samara.suplici@gmail.com

Recebido em 02/03/2020.

Aprovado em 21/06/2021.

\section{RESUMO}

Objetivo: elaborar um modelo interpretativo sobre a adesão ao autocuidado de pessoas com Diabetes Mellitus na Atenção Primária à Saúde. Método: estudo de método misto realizado em quatro Unidades Básicas de Saúde. O estudo quantitativo transversal foi composto por 329 participantes, elegeram-se variáveis sociodemográficas e clínicas e o questionário de atividades de autocuidado. No estudo qualitativo, com 31 participantes, utilizou-se a vertente construtivista da Teoria Fundamentada nos Dados. Os dados foram analisados utilizando-se a estatística descritiva e a codificação inicial e focalizada. Resultados: a maioria dos participantes é do sexo feminino, casada, com um a cinco anos de estudo, aposentada e com renda de um a três salários-mínimos. Obteve-se baixa adesão à alimentação saudável, à atividade física e ao monitoramento glicêmico. A adesão desejável foi relacionada ao uso de medicamentos e cuidados com os pés. Na etapa qualitativa, obteve-se como fenômeno: Enfrentando a complexidade do tratamento do Diabetes Mellitus e buscando o autocuidado. Este foi sustentado por duas categorias: Encontrando as dificuldades para seguir o tratamento e Valorizando os aspectos facilitadores do tratamento que deram subsídios para a elaboração do modelo. Conclusões e implicações para a prática: foi elaborado um modelo interpretativo cujos elementos demonstram a complexidade do fenômeno e contribuem para a adesão ao autocuidado nessa população.

Palavras-chave: Autocuidado; Diabetes Mellitus; Atenção Primária à Saúde; Cooperação e Adesão ao Tratamento; Teoria Fundamentada.

\section{Abstract}

Objective: to develop an interpretive model on the adherence to self-care of people with Diabetes Mellitus in Primary Health Care. Method: a mixed-method study conducted in four Basic Health Units. The cross-sectional quantitative study was composed of 329 participants, sociodemographic and clinical variables and the questionnaire of self-care activities were chosen. In the qualitative study, with 31 participants, the constructivist strand of Grounded Theory was used. The data were analyzed using descriptive statistics and initial and focused coding. Results: most participants are female, married, with one to five years of schooling retired, and with an income of one to three minimum wages. Low adherence to healthy eating, physical activity, and glycemic monitoring was obtained. The desirable adherence was related to the use of medications and foot care. In the qualitative step the phenomenon was: Facing the complexity of the treatment of Diabetes Mellitus and seeking self-care. This was supported by two categories: Encountering difficulties to follow the treatment and valuing the facilitating aspects of the treatment that provided subsidies for the development of the model. Conclusions and implications for practice: an interpretative model was elaborated whose elements demonstrate the complexity of the phenomenon and contribute to the adherence to self-care in this population.

Keywords: Self Care; Diabetes Mellitus; Primary Health Care; Treatment Adherence and Compliance; Grounded Theory.

\section{REsumen}

Objetivo: elaborar un modelo interpretativo sobre adhesión al autocuidado de personas con Diabetes Mellitus en Atención Primaria de Salud. Método: estudio de método mixto realizado en cuatro Unidades Básicas de Salud. El estudio cuantitativo transversal fue compuesto por 329 participantes, se eligieron variables sociodemográficas y clínicas y el cuestionario de actividades de autocuidado. En el estudio cualitativo, con 31 participantes, se utilizó el enfoque constructivista de Grounded Theory. Los datos se analizaron mediante estadística descriptiva y codificación inicial y focalizada. Resultados: la mayoría de los participantes son mujeres, casadas, con uno a cinco años de estudio, jubiladas y con ingresos de uno a tres salarios mínimos. Se obtuvo una baja adhesión a la alimentación saludable, la actividad física y el control glucémico. La adhesión deseable se relacionó con el uso de medicamentos y el cuidado de los pies. En la etapa cualitativa se obtuvo el siguiente fenómeno: Enfrentando la complejidad del tratamiento de la Diabetes Mellitus y buscando el autocuidado. Esto fue apoyado por dos categorías: Encontrando las dificultades para seguir el tratamiento y Valorando los aspectos facilitadores del tratamiento que apoyaron el desarrollo del modelo. Conclusiones e implicaciones para la práctica: se desarrolló un modelo interpretativo cuyos elementos demuestran la complejidad del fenómeno y contribuyen a la adhesión al autocuidado en esta población.

Palabras clave: Autocuidado; Diabetes Mellitus; Atención Primaria a la Salud; Cooperación y Adhesión al Tratamiento; Teoría Fundamentada. 


\section{INTRODUÇÃO}

O autocuidado refere-se à capacidade da própria pessoa em realizar ações que visam à preservação da sua saúde, desenvolvimento e bem-estar ${ }^{1}$. O autocuidado de pessoas com Diabetes Mellitus do tipo 2 (DM2) implica mudanças no estilo de vida que são necessárias para prevenir complicações decorrentes da doença que podem levar à incapacidade ou morte. Manter uma alimentação saudável, atividade física regular, uso contínuo de medicamentos, verificação frequente da glicemia e cuidados com os pés estão entre as atividades necessárias para a cooperação e a adesão ao tratamento que garantem a manutenção do autocuidado de pessoas com DM2 ${ }^{1,2}$.

$A$ adesão ao autocuidado pode mudar como resultado de circunstâncias inerentes à própria pessoa ${ }^{3,4}$ e questões externas que envolvem aspectos sociais ${ }^{5}$ e ações realizadas pelo sistema de saúde ${ }^{6,7}$. Nesse sentido, a Atenção Primária à Saúde (APS) tem caráter estratégico para a promoção do autocuidado por meio de um cuidado integral e resolutivo às pessoas com DM2.

As decisões diárias que as pessoas com DM2 precisam tomar para manter o autocuidado envolvem questões que vão muito além de uma resolução individual ${ }^{3,8} \mathrm{e}$, por isso, a compreensão dos problemas não pode ser analisada isoladamente ou sob um único foco, mas esses devem ser interpretados dentro de um olhar sistêmico, ou seja, interdependentes de um contexto ${ }^{9}$.

Nesse sentido, ao considerar que a adesão ao autocuidado de pessoas com DM2 envolve múltiplos fatores interdependentes, este estudo teve embasamento teórico no Pensamento Complexo de Edgar Morin ${ }^{9}$. Essa teoria permite compreender as diversas dimensões da realidade, colocando o fenômeno inseparável do contexto histórico e social. A complexidade engloba ações, relações e interações e, assim, não incorre na contradição da simplificação e redução do fenômeno ${ }^{9}$.

Apesar de estudos evidenciarem que a adesão ao autocuidado pode ser influenciada por aspectos individuais, sociais e do sistema de saúde ${ }^{3,4,6,7}$,encontra-se uma lacuna do conhecimento referente às relações e interações entre estes aspectos no autocuidado. Pressupõe-se que a adesão ao autocuidado de pessoas com DM2 atendidas na APS seja um fenômeno complexo no qual existam interações entre múltiplos fatores individuais, sociais e do sistema de saúde. Nesta interdependência, emergem as questões norteadoras: (1) Como vem ocorrendo a adesão ao autocuidado de pessoas com DM2 atendidas na APS? (2) Quais as relações e interações envolvidas na adesão ao autocuidado de pessoas com DM2 atendidas na APS?

O objetivo foi elaborar um modelo interpretativo sobre a adesão ao autocuidado de pessoas com DM no contexto da APS.

\section{MÉTODO}

Trata-se de pesquisa de métodos mistos, do tipo sequencial explanatório ${ }^{10}$, realizada em duas etapas. A primeira consistiu em um estudo descritivo, com abordagem quantitativa, redigido conforme recomendação STROBE, implementado primeiro e com menor ênfase. A segunda etapa foi uma pesquisa qualitativa que utilizou a vertente construtivista da Teoria Fundamentada nos Dados (TFD), proposta por Charmaz ${ }^{11}$, para explicar os resultados quantitativos. A escolha da vertente construtivista da TFD adequa-se ao estudo da adesão ao autocuidado de pessoas com DM2 na medida em que esta reconhece os fenômenos estudados como construções cujos preceitos respondem à tradição interpretativa ${ }^{11}$. Essa etapa foi redigida conforme recomendação COREQ.

O estudo foi realizado em quatro Unidades Básicas de Saúde (UBS) do município de São José, Santa Catarina, Brasil, nos meses de janeiro a junho de 2017. Essas unidades foram eleitas devido ao vínculo e à experiência da pesquisadora com essas unidades do município e interesse dos gestores locais.

A população foi constituída por 1.285 pessoas com DM2 cadastradas nas quatro UBS. Elegeram-se como critérios de inclusão: usuários dos serviços de saúde com idade superior a 18 anos e com diagnóstico autorreferido de DM2 há mais de um ano. Elencaram-se como critérios de exclusão: pessoas acamadas, sem condições de realizar as atividades de autocuidado constantes no instrumento de coleta de dados. Para a primeira etapa, denominada estudo de abordagem quantitativa, foi calculado, a partir da população, o tamanho mínimo da amostra de 296 pessoas, com estimativa de $50 \%$ e intervalo de confiança de $95 \%$. Desse modo, a amostra intencional deste estudo foi de 329 pessoas conforme a população de pessoas com DM2 cadastradas em cada UBS (Tabela 1).

Para este estudo, elegeram-se como variáveis sociodemográficas: estado civil (casado, solteiro, separado/divorciado, viúvo); faixa etária (até 59 anos, 60-69 anos, 70 anos ou mais); sexo (masculino, feminino); renda em salários-mínimos (menos de um, de um a três, de três a seis, mais de seis); anos de escolaridade (nunca estudou, de um a cinco anos, de seis a nove anos, de dez a 12 anos, 13 anos ou mais). As variáveis clínicas foram: presença de comorbidades; tempo de doença (de um a cinco anos, de seis a dez anos, de 11 a 20 anos, mais de 20 anos) e o tipo de tratamento (hipoglicemiantes orais, hipoglicemiantes orais + insulinoterapia, insulinoterapia). Os dados foram obtidos por meio de instrumento estruturado.

As variáveis referentes ao autocuidado foram avaliadas por meio do Questionário de Atividades de Autocuidado com o Diabetes (QAD), versão validada do instrumento Summary of Diabetes Self-Care Activities (SDSCA). O instrumento é uma medida de autorrelato que avalia seis dimensões dos comportamentos de autocuidado referentes aos últimos sete dias: alimentação geral (dois itens); alimentação específica (três itens); atividade física (dois itens); monitorização da glicemia (dois itens); cuidados com os pés (três itens); uso da medicação (três itens, sendo que a pessoa responde um dos itens de acordo com o esquema medicamentoso). A avaliação é padronizada em dias da semana. As respostas são pontuadas de zero a sete conforme seja menos desejável (zero) e mais desejável (sete) ${ }^{12}$. Como o QAD não propõe um ponto de corte para a adesão desejável, foi considerado, neste estudo, baseado em estudo anterior $^{13}$, que o mais desejável é que a pessoa tenha seguido 
Tabela 1. Frequências absoluta e relativa dos participantes do estudo por Unidade Básica de Saúde. São José, SC, Brasil, 2017.

\begin{tabular}{cccc}
\hline UBS & $\begin{array}{c}\text { Número de } \\
\text { pessoas com DM } \\
\text { cadastradas }\end{array}$ & $\mathrm{N}$ & $\%$ \\
\hline $\mathbf{1}$ & 387 & 99 & 30,1 \\
\hline $\mathbf{2}$ & 201 & 51 & 15,5 \\
\hline $\mathbf{3}$ & 356 & 91 & 27,7 \\
\hline $\mathbf{4}$ & 341 & 88 & 26,7 \\
\hline TOTAL & 1.285 & 329 & 100.0 \\
\hline
\end{tabular}

Legenda: $n$ = Frequência absoluta; \% = Frequência relativa. Fonte: dados da pesquisa.

as recomendações de cada um dos itens durante, pelo menos, cinco dias da semana. Assim, foram consideradas como mais desejável de 5-7 dias e como menos desejável 0-4 dias. Para os subitens consumo de gorduras e consumo de doces, os valores foram invertidos conforme preconizado no instrumento, sendo considerado mais desejável de 0-2 dias e menos desejável de 3-7 dias. Os hábitos tabágicos também são investigados no instrumento, mas não foram abordados neste estudo.

Os participantes foram convidados por cartas entregues às pessoas com DM2 pelos Agentes Comunitários de Saúde (ACS) e aqueles que aceitaram participar foram agendados para a aplicação dos instrumentos de coleta de dados (questionário contendo as variáveis sociodemográficas e clínicas e QAD). Os participantes foram esclarecidos sobre a pesquisa e o Termo de Consentimento Livre e Esclarecido foi lido e assinado. $O$ questionário e o QAD foram aplicados individualmente na UBS ou na residência da pessoa pela pesquisadora principal e/ou por uma aluna do curso de Graduação em Enfermagem treinada previamente pela pesquisadora principal. $O$ tempo médio de cada questionário foi de 15 minutos.

Os dados foram organizados no Excel $^{\circledR} \mathrm{e}$ as análises foram realizadas por meio do software IBM SPSS (versão 20.0). Na análise descritiva, foram verificadas as frequências absoluta e relativa (variáveis categóricas) e as medidas de posição e dispersão dos dados (variáveis numéricas).

A segunda etapa, denominada estudo de abordagem qualitativa, foi guiada pelo estudo quantitativo desde a escolha dos participantes até a elaboração das questões norteadoras. De acordo com o estabelecido na TFD, os participantes foram elencados por meio da composição de grupos amostrais direcionados por hipóteses que surgiram a partir das análises. Por isso, participaram dessa etapa, além de pessoas com DM2, profissionais de saúde que atuam no cuidado a estas pessoas, processo mais bem detalhado a seguir. Foi uma amostragem intencional na qual as pessoas com DM2 receberam o convite ainda na etapa quantitativa, sendo critério de inclusão ter participado da etapa anterior. Quanto aos profissionais de saúde, o critério de inclusão foi ter mais de 18 anos e trabalhar há mais de um ano no cuidado às pessoas com DM2 na APS.

A partir dos resultados do estudo quantitativo, emergiu a hipótese de que as pessoas com DM2 que aderem ao autocuidado encontram significados para fazê-lo. Isso direcionou a formação do primeiro grupo amostral composto por doze pessoas com DM2 que apresentaram adesão ao autocuidado mensurada pelo QAD. Esse grupo foi identificado pela letra $E$ de entrevistado, seguida pelo número da entrevista de um a 12 (E1 a E12). Após as análises dessas entrevistas, chegou-se à hipótese de que poderia existir uma diferença no significado do autocuidado entre aqueles que não apresentaram adesão ao autocuidado mensurada pelo QAD, fato que definiu o segundo grupo amostral composto por doze pessoas com DM2. Esse grupo foi identificado de E13 a E24. Para o primeiro e segundo grupos amostrais, a coleta dos dados teve como questão inicial: "Fale-me sobre o significado do autocuidado em Diabetes para você".

$\mathrm{Na}$ análise das entrevistas do segundo grupo, evidenciou-se a hipótese de que a APS é um apoio na adesão ao autocuidado, a qual gerou outros questionamentos. Para buscar dados que corroborassem ou redirecionassem tal hipótese, foi composto o terceiro grupo amostral, formado por sete profissionais da APS, que teve como questão: "Fale-me sobre o significado do cuidado prestado por você às pessoas com DM2 referentes à promoção do autocuidado". Esse grupo foi identificado pela letra P seguida pelo número da entrevista (P25 a P31). Ao final dessas análises, julgou-se chegar à amostragem teórica e à saturação das categorias.

A saturação foi definida quando não foram encontrados mais dados pelos quais se pudessem desenvolver propriedades da categoria, ou seja, quando ocorreram instâncias semelhantes repetidamente, fazendo com que houvesse a necessidade de procurar grupos que estendessem a diversidade de dados 0 máximo possível, garantindo que a saturação fosse baseada na maior variedade possível de dados sobre a categoria ${ }^{14}$.

$\mathrm{Na}$ coleta de dados, utilizaram-se a observação e as entrevistas em profundidade. A observação consistiu em detectar situações reais vivenciadas pela pesquisadora que produzissem reflexões acerca do fenômeno. As observações foram anotadas diariamente. As entrevistas foram realizadas pela pesquisadora principal individualmente em consultório das UBS ou na residência da pessoa e duraram, em média, 40 minutos. Foram audiogravadas e transcritas.

Os documentos oriundos das observações e entrevistas foram exportados para o software Atlas Ti, versão 1.0.45 para Mac (licença n.110271462), no qual foi realizado o processo de codificação e organização dos dados. As análises ocorreram simultaneamente com a coleta de dados como preconizado na TFD. Utilizou-se uma codificação inicial, na qual cada segmento de dado foi codificado no software, seguida por uma fase seletiva e focalizada na qual os códigos iniciais mais significativos ou frequentes foram classificados, integrados, sintetizados e organizados em categorias e subcategorias, orientados pela proposta de Charmaz ${ }^{11}$. 
A pesquisa seguiu os aspectos éticos da Resolução $\mathrm{n}^{\circ}$ 466/2012 do Conselho Nacional de Saúde (CNS), sendo aprovada pelo Comitê de Ética em Pesquisa da Universidade Federal de Santa Catarina (UFSC).

\section{RESULTADOS}

Quanto às características sociodemográficas e clínicas, dos $329(100 \%)$ participantes, a maioria era do sexo feminino $(71,70 \%)$, na faixa etária de 60 a 69 anos (38,60\%), casada (50,50\%), com um a cinco anos de estudo (48,60\%), aposentada $(58,70 \%)$, com renda familiar de um a três salários-mínimos $(73,20 \%)$, sendo que o salário-mínimo vigente em 2017 era de $\mathrm{R} \$ 937,00$. As comorbidades mais prevalentes foram a hipertensão arterial (78,70\%), a dislipidemia $(55,6 \%)$ e a doença cardíaca $(30,1 \%)$. A maioria tinha de um a cinco anos de diagnóstico para o DM2 $(65.6 \%)$ e utilizava apenas os hipoglicemiantes orais como terapia medicamentosa $(65,9 \%)$.

No que se refere à adesão às várias dimensões do autocuidado, as pessoas com DM2 que apresentaram comportamentos de autocuidado desejáveis estão apresentados a seguir (Tabela 2).

Dentre as características dos participantes da segunda etapa, destaca-se que, dos 12 participantes do primeiro grupo amostral, nove eram do sexo feminino, a idade variou de 61 a 83 anos e o tempo de doença, de dois a 40 anos. No segundo grupo, entre os 12 participantes, dez eram do sexo feminino, a idade variou de 38 a 90 anos e o tempo de doença entre dois e 20 anos. No terceiro grupo amostral, dentre os sete participantes, duas eram enfermeiras, dois, educadores físicos, duas, Agentes Comunitárias de Saúde (ACS) e uma, médica, a idade variou entre 33 e 55 anos e o tempo de formação profissional, entre três e 30 anos.

A partir do processo analítico guiado pela TFD, obteve-se como fenômeno: Enfrentando a complexidade do tratamento do Diabetes Mellitus e buscando o autocuidado. Esse fenômeno foi sustentado por duas categorias construídas a partir da comparação e triangulação dos dados entre os três grupos amostrais. As categorias encontradas estão apresentadas no Quadro 1.

A categoria encontrando as dificuldades para seguir o tratamento mostra a multidimensionalidade das dificuldades encontradas no autocuidado.

A percepção dos aspectos individuais como dificultadores do autocuidado refere-se aos hábitos adquiridos durante toda uma vida, principalmente na adesão a uma dieta saudável e à prática regular de atividade física. $\mathrm{O}$ fato de a doença ser

Tabela 2. Frequência dos desfechos desejáveis referentes às atividades de autocuidado de pessoas com Diabetes Mellitus do tipo 2. São José, SC, Brasil, 2017 ( $n=329)$.

\begin{tabular}{|c|c|c|}
\hline Atividades de autocuidado do QAD & $\mathrm{F}$ & $\%$ \\
\hline \multicolumn{3}{|l|}{ Alimentação Geral } \\
\hline 1. Seguiu uma dieta saudável (5-7 dias/sem) & 156 & 47,4 \\
\hline 2. Seguiu a orientação alimentar dada por profissional de saúde (5-7 dias/sem) & 153 & 46,6 \\
\hline \multicolumn{3}{|l|}{ Alimentação Específica } \\
\hline 1. Ingeriu cinco ou mais porções de frutas e/ou vegetais (5-7 dias/sem) & 198 & 60,1 \\
\hline 2. Ingeriu alimentos ricos em gordura $(0-2$ dias $/ \mathrm{sem}) *$ & 231 & 70,3 \\
\hline 3. Ingeriu doces (0-2 dias/sem)* & 241 & 73,3 \\
\hline \multicolumn{3}{|l|}{ Atividade Física } \\
\hline 1. Realizou atividade física por, pelo menos, $30 \mathrm{~min}$ (5-7 dias/sem) & 50 & 15,3 \\
\hline 2. Realizou exercício físico específico (5-7 dias/sem) & 44 & 13,5 \\
\hline \multicolumn{3}{|l|}{ Monitorização da Glicemia } \\
\hline 1. Avaliou o açúcar no sangue (5-7 dias/sem) & 85 & 25,8 \\
\hline 2. Avaliou o açúcar no sangue o número de vezes recomendado por profissional (5-7 dias/sem) & 80 & 24,4 \\
\hline \multicolumn{3}{|l|}{ Cuidados com os pés } \\
\hline 1. Examinou os pés (5-7 dias/sem) & 246 & 74,8 \\
\hline 2. Examinou os sapatos antes de calçá-los (5-7 dias/sem) & 232 & 70,8 \\
\hline 3. Secou os espaços entre os dedos dos pés depois de lavá-lo (5-7 dias/sem) & 276 & 83,9 \\
\hline \multicolumn{3}{|l|}{ Medicação } \\
\hline Tomou os medicamentos conforme recomendado (5-7 dias/sem) & 276 & 83,9 \\
\hline
\end{tabular}

* Escore reverso

Fonte: dados da pesquisa. 
Quadro 1. Categorias e subcategorias do fenômeno "Enfrentando a complexidade do tratamento do Diabetes Mellitus e buscando o autocuidado". São José, SC, Brasil, 2017.

\begin{tabular}{|ll|}
\hline \multicolumn{1}{|c|}{ Categorias } & \multicolumn{1}{c|}{ Subcategorias } \\
\hline $\begin{array}{l}\text { Encontrando as dificuldades para } \\
\text { seguir o tratamento }\end{array}$ & $\begin{array}{l}\text { Percebendo aspectos individuais como dificultadores do autocuidado } \\
\text { Vivendo em uma sociedade marcada pela determinação social da doença }\end{array}$ \\
\hline $\begin{array}{l}\text { Valorizando os aspectos } \\
\text { facilitadores do tratamento }\end{array}$ & Sercebendo-se vulnerável diante da Atenção Primária à Saúde \\
& Contornando as dificuldades sociais e enfrentando o tratamento \\
\hline
\end{tabular}

Fonte: elaborado pelos autores.

silenciosa, a dificuldade de relacionar as alterações da glicemia com as práticas diárias, questões emocionais, o efeito colateral das medicações e a presença de outros problemas de saúde, às vezes, considerados mais graves que o DM2 dificultam a adesão à dieta saudável e à atividade física.

Fui acostumada a comer besteira, a gente comia só salgado (E15). Não fui acostumada a fazer atividade física. Então, acho muito difícil incorporar esse hábito (E14). As pessoas não dão o devido valor [...] pro problema que é silencioso, a pessoa não sente nada naquele momento, e ela vai levando (P27).

As pessoas com DM2 reconhecem viver em uma sociedade marcada pela determinação social da doença. As dificuldades financeiras, as questões familiares e os problemas na esfera política local e global foram apontados como dificultadores do tratamento e refletem-se na compra do alimento, dos medicamentos, na realização de atividade física e ainda na realização de exames ou consultas. Os profissionais de saúde também reconhecem que os aspectos sociais interferem no autocuidado em Diabetes e que medidas do sistema de saúde podem minimizar essas questões.

Porque, quando eu posso comprar uma fruta, eu merendo uma fruta, eu sei o que é bom pra mim, mas não dá pra comprar sempre (E18). E outros tipos de atividades seria bom, tipo uma hidro, mas não dá para mim, é caro! (E13). Nós trabalhamos com uma população que tem muitos problemas e a gente tem que saber como lidar senão eles abandonam o tratamento pra cuidar de outra questão (P26). Eu tenho trabalhado com o grupo de exercícios físicos com exercícios e materiais que os pacientes trazem de casa pra que eles possam repetir esses exercícios em casa (P27).

Pela observação realizada nos domicílios, a determinação social da doença pôde ser percebida na precariedade das condições de vida, fazendo com que essas pessoas fiquem em uma situação de vulnerabilidade social. Assim, outros problemas sociais, considerados maiores que o DM2, fazem as pessoas adiarem o tratamento para dedicarem-se a questões consideradas mais urgentes.

Ficou evidenciado ainda que as pessoas com DM2 dos grupos amostrais 1 e 2 percebem-se vulneráveis diante da APS. A demora na realização dos exames, a falta insumos e de medicamentos básicos e/ou mais eficazes para o controle glicêmico, a dificuldade de encaminhamento ao especialista, a mudança constante de médicos, a falta de fitas reagentes e de lancetas para o controle glicêmico e as questões burocráticas do serviço de saúde foram vistas como dificultadoras para o autocuidado.

Para fazer um exame, demora, meu endócrino já faz tempo que não consigo consultar[...] deixa a gente meio ruim, o fato de chegar lá (UBS) e não ter o remédio (E07). Eu tinha que fazer o teste (glicemia) três vezes por semana, tenho pedido da médica para fazer no posto, até comecei certo, mas o posto daqui fica longinho, difícil se programar (E15). Ainda está faltando fita e lanceta, esse mês, fui obrigado a comprar, mas é caro demais (E08).

Os profissionais de saúde reconhecem que questões burocráticas relacionadas ao sistema de saúde e a relação com os profissionais interferem no autocuidado. Mas as opiniões sobre a interferência da falta de glicosímetros e fitas reagentes, da dificuldade de exames e de encaminhamento para os especialistas divergem daquelas apresentadas pelas pessoas com DM2.

Se é rotina, vai esperar até três meses (os exames). Agora, se está com alguma alteração, a gente consegue liberar antes. Porque aqui a gente libera exames de acordo com os dados clínicos (P26). A gente precisa tirar aquela questão de que tudo é na base do encaminhamento que está muito enraizado. As pessoas não entenderam o que é realmente essa questão da Saúde da Família (P28). 
A superação dessas dificuldades foi evidenciada entre as pessoas com DM2 que aderem ao tratamento. Assim, obteve-se a categoria valorizando os aspectos facilitadores do tratamento, que evidenciou diferenças entre os dois grupos amostrais de pessoas com DM2. Quanto aos profissionais de saúde, estes reconhecem que a valorização dos aspectos facilitadores em detrimento das dificuldades é um importante aspecto ligado ao autocuidado.

Tanto as pessoas com DM2 que aderem ao tratamento como aquelas que não aderem reconhecem os aspectos facilitadores, mas eles parecem ser mais valorizados entre aqueles que aderem ao tratamento. Por isso, estes últimos destacam que superar as dificuldades individuais e aceitar o tratamento são fundamentais para o autocuidado. A aceitação da doença, o enfrentamento do tratamento com pensamento positivo e encontrar apoio em si mesmo são aspectos mencionados.

E depois que eu comecei a aceitar, a glicose está normal, então, é preciso aceitar e aprender a conviver com ela (E06). Meu apoio sou eu mesma, porque sei que não vou ficar boa, mas tomando o remédio, fazendo dieta, vou viver bem, buscando uma vida melhor (E08).

Entre aqueles que não aderem ao tratamento, existe uma dificuldade de aceitação ou uma não valorização da doença e do tratamento e também um reconhecimento de que falta uma motivação dentro de si para o enfrentamento da doença e até uma culpabilização de si mesmo.

Não me preocupo com ela! Tento viver como se nada estivesse acontecendo, não tenho doença nenhuma (E16). Acho que falta força de vontade minha. Eu sei como deve ser, mas não faço! [...] sinceramente, não tenho apoios. Mas acho que depende de mim mesma (E24).

Os profissionais de saúde também reconhecem ser necessária a superação das dificuldades individuais, sendo a própria pessoa a principal responsável pelo tratamento, mas que apoios sociais e do sistema de saúde favorecem o autocuidado.

A participação da pessoa no tratamento é importante. No caso da atividade física, a gente está tentando remediar. Porque eles não tiveram essa educação e quando entendem a importância, começam a praticar. Então, depende deles, lógico, mas depende de muitos outros fatores educacionais (P28).

No contexto social, contornar as dificuldades sociais e enfrentar o tratamento foram vistos como um dos facilitadores do tratamento. Isso significa possuir recursos financeiros, encontrar apoio na família e em programas públicos ou espaços públicos.
Eu vou a médico sempre, faço meus exames, de seis em seis meses, tem que ser pelo plano, não dá pra esperar o SUS (E02). Acho o CAT (Centro de Atenção à Terceira Idade) uma economia pro município, as pessoas que frequentam são menos doentes justamente pela atividade e pelo interagir (E05). O importante é a rede de apoio social. O Serviço Social deveria estar junto porque o problema de quase tudo tem alguma coisa social (P26).

No contexto da população estudada, muitos aspectos facilitadores do tratamento foram encontrados dentro do sistema de saúde. As pessoas com DM2 reconhecem a APS como apoio e buscam o autocuidado.

Depois que eu comecei a frequentar mais o posto, eu vejo que a gente só aprende as coisas se estiver mais próximo. Eu preciso procurar mais o médico, buscar o que eu posso fazer para cuidar mais de mim (E07). Sempre que eu tenho dúvida, eu procuro a (enfermeira da área), ela me orienta sobre a alimentação, sobre os cuidados com a pele, com os pés. Eu não sabia que o problema da pessoa, às vezes, começa no pé (E12). Manternosso grupo de atividade física depende muito da colaboração da equipe, da coordenadora do posto (P28).

As falas acima apontam que, no que se refere ao apoio da APS, se destacaram: o vínculo estabelecido com os profissionais de saúde; a importância do trabalho dos enfermeiros, educadores físicos e médicos; os grupos de atividade física ou de hábitos saudáveis; a referência das enfermeiras das áreas e a proximidade dos ACS como esclarecedores de dúvidas relacionadas à doença e ao tratamento e também sobre o funcionamento do SUS. Isso também foi evidenciado durante a observação quando, a caminho das entrevistas, muitas vezes, os ACS eram abordados pela população, que perguntava sobre dúvidas clínicas ou burocráticas do serviço, sendo que estes davam os encaminhamentos necessários.

A Figura 1 apresenta o modelo interpretativo que ilustra a articulação entre as dimensões do autocuidado das pessoas com DM2, as categorias apresentadas e o fenômeno do estudo.

\section{DISCUSSÃO}

A etapa quantitativa evidenciou uma baixa adesão à dieta geral, à atividade física e à monitorização glicêmica. Uma adesão mais desejável foi evidenciada na alimentação específica, nos cuidados com os pés e no uso da medicação. Esses achados são semelhantes aos apresentados em outros estudos que também evidenciaram baixa adesão à dieta saudável ${ }^{8,15}$, à atividade física ${ }^{8,16,17}$ e ao monitoramento da glicose ${ }^{8,15}$. Com relação aos cuidados com os pés, bons níveis de autocuidado foram encontrados ${ }^{8}$. Uma melhor adesão ao uso da medicação também tem sido encontrada nos estudos $8,15,16,18$. 


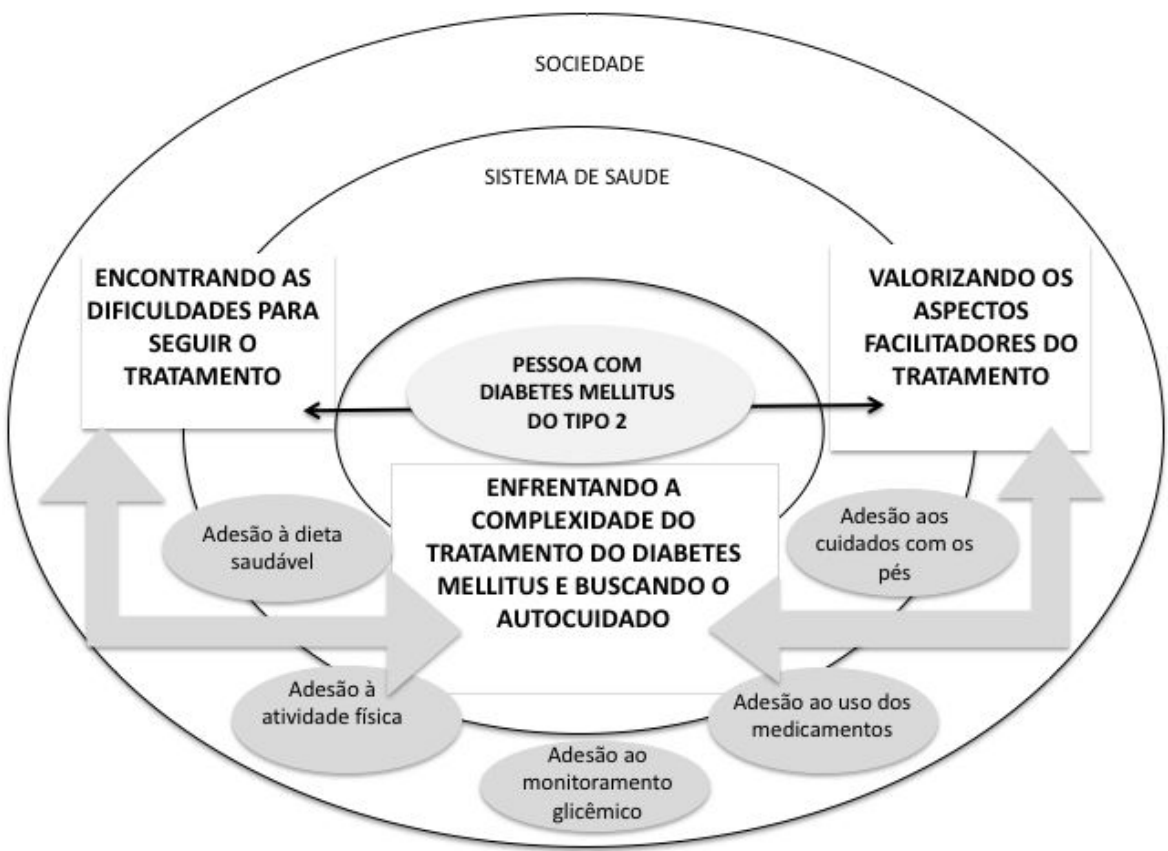

Figura 1. Diagrama representativo da articulação entre as dimensões do autocuidado das pessoas com DM2, as categorias e o fenômeno. São José, SC, Brasil, 2017.

Fonte: elaborado pelos autores.

A etapa qualitativa evidenciou que as dificuldades encontradas para seguir o tratamento são comuns entre as pessoas que aderem e aquelas que não aderem ao tratamento. Superar essas dificuldades parece ser a diferença entre aderir e não aderir ao autocuidado.

Desse modo, na triangulação dos resultados quantitativos e qualitativos, houve uma convergência. Assim, a segunda etapa explica alguns dados da primeira etapa da pesquisa. No que se refere à adesão às várias dimensões do autocuidado entre as pessoas com DM2, a categoria encontrando as dificuldades para seguir o tratamento explica as baixas prevalências de adesão desejável à dieta saudável, atividade física e monitorização glicêmica encontradas na etapa quantitativa. As dificuldades apontadas para a adesão à dieta e à atividade física foram mais localizadas nos âmbitos individual e social. Nestas duas dimensões do autocuidado, convergem, entre os três grupos amostrais, a dificuldade de mudança dos hábitos adquiridos durante a vida, as dificuldades financeiras e a falta de apoio da família ou rede social.

De fato, a mudança de hábitos adquiridos durante a vida é um importante componente para a adesão à dieta e à atividade física. Uma revisão integrativa apontou que pessoas com DM2 que praticavam hábitos saudáveis antes do aparecimento da doença relataram maior facilidade de adesão a uma dieta saudável e à atividade física ${ }^{19}$. Outros estudos evidenciaram ainda que a motivação intrínseca e o enfrentamento da doença com pensamento positivo têm relação com a adesão à atividade física $^{4,20}$. Quanto às dificuldades financeiras, as pessoas com DM2 têm a convicção de que um melhor poder aquisitivo permite a promoção de uma dieta adequada à sua condição de saúde ${ }^{5}$. A falta de apoio da família também pode ser um dificultador na adesão a estas dimensões do autocuidado ${ }^{19}$.

Apesar das baixas prevalências de adesão à atividade física, destaca-se, entre aqueles que aderem a essa dimensão do autocuidado, que muitos aspectos facilitadores estão nos âmbitos social e do sistema de saúde. Nesse sentido, espaços sociais como o Centro de Atenção à Terceira Idade (CAT), espaços públicos destinados à prática de atividade física e os grupos de atividades físicas em algumas UBS foram mencionados.

A importância de espaços públicos para a prática de atividades físicas vem sendo reconhecida pelas políticas públicas de saúde e, em face disso, há que se mencionar o Programa Academias da Saúde, visando à implantação de polos com infraestrutura, equipamentos e profissionais para a orientação de práticas de atividade física e modos de vida saudáveis de forma coordenada com os Núcleos Ampliados de Saúde da Família (NASF)21. Os esforços conjuntos que vêm sendo feitos para manter grupos de atividades físicas continuam em algumas UBS e envolvem equipe médica, de Enfermagem e educadores físicos. A literatura aponta que as atividades físicas em grupo, com suporte de profissionais, mostram-se como uma importante estratégia para a adesão a essa dimensão do autocuidado ${ }^{22}$.

Quanto à adesão ao monitoramento glicêmico, as baixas prevalências de adesão desejável encontradas podem também ser explicadas na categoria encontrando as dificuldades para seguir o tratamento, sendo, neste caso, mais relacionadas ao sistema de saúde. A falta de fitas reagentes para uso no glicosímetro faz com que as pessoas com DM2 busquem, às vezes, estratégias 
equivocadas como a não verificação conforme recomendado pelos profissionais. Os profissionais, por sua vez, reconhecem que estes dispositivos são importantes no tratamento, mas outros aspectos são igualmente importantes.

Inegavelmente, a monitorização glicêmica tem um papel importante no autocuidado entre as pessoas com DM2, no entanto, a monitorização sozinha não diminui os níveis de glicose no sangue. Para ser útil, as informações devem ser integradas em planos clínicos e de autocuidado ${ }^{2}$, o que foi muito enfatizado pelos profissionais de saúde neste estudo.

No que se refere aos cuidados com os pés, a prevalência de adesão mais desejável pode ser explicada pela categoria valorizando os aspectos facilitadores do tratamento, que evidencia a importância dos profissionais nas orientações referentes a essa dimensão do autocuidado. A prevalência de pessoas que cuidam dos pés em uma frequência mais desejável pode ser resultado dessas práticas de orientação. Um estudo realizado no Nordeste brasileiro encontrou associação entre o nível de conhecimento e a maior adesão aos cuidados com os pés e destacou a importância de os profissionais de saúde, em especial, dos enfermeiros, realizarem orientações quanto aos cuidados que devem ser praticados ${ }^{13}$.

O uso da medicação em uma frequência desejável na população estudada também pode ser resultado das ações do sistema de saúde. A Política Nacional de Assistência Farmacêutica e a criação de programas públicos para a obtenção dos medicamentos em sistema de copagamento em farmácias públicas ou privadas credenciadas atuam sobre as barreiras de acesso financeira e geográfica ${ }^{23}$. $\mathrm{O}$ acesso à medicação é uma importante estratégia de adesão à terapia medicamentosa ${ }^{24,25}$.

No entanto, foram mencionadas muitas dificuldades referentes à disponibilidade de medicamentos e, principalmente, à falta de medicamentos mais eficazes, que, muitas vezes, não são fornecidos pelo sistema público. Um estudo que avaliou os fatores associados à adesão terapêutica na APS no Norte do Brasil evidenciou que a dificuldade de conseguir os medicamentos interfere na adesão a essa dimensão do autocuidado ${ }^{24}$.

Ficou evidenciado que a adesão às várias dimensões do tratamento envolve a superação de dificuldades e a valorização de aspectos facilitadores do tratamento nos âmbitos individual, social e do sistema de saúde.

Outros estudos enfatizaram que os fatores individuais são responsáveis pela melhor adesão ao autocuidado ${ }^{4}$. $O$ apoio social encontrado na família, nos recursos financeiros ou nos programas públicos direcionados à saúde também foram aspectos sociais facilitadores para o autocuidado mencionados neste estudo. Isso está em consonância com o resultado de outro estudo realizado com pessoas com DM2 atendidas na APS no Sul do Brasil. Esse estudo evidenciou que o apoio social exerce influência sobre os comportamentos de autocuidado em DM2, sendo que o apoio da família foi relacionado à alimentação mais saudável e à realização de atividade física mais frequentemente ${ }^{19}$. Outro estudo realizado na região Centro-Oeste brasileira também apontou a falta de apoio familiar como um dificultador na adesão ao autocuidado ${ }^{25}$.
A APS foi apontada como um apoio para as pessoas com DM2. Apesar das dificuldades estruturais e organizacionais evidenciadas, as relações humanas estão mantidas e as pessoas confiam na APS e nos profissionais. Existem evidências na literatura de que a APS tem importante papel no autocuidado em pessoas com DM2, uma vez que os profissionais da APS atuam como agentes facilitadores e mobilizadores por meio da conscientização, mudança de comportamento e desenvolvimento da capacidade e habilidade do indivíduo para o autocuidado ${ }^{7,26}$.

Ao analisar esses resultados sob a ótica do pensamento complexo, pode-se afirmar que $o$ autocuidado entre as pessoas com DM2 é um fenômeno complexo e multidimensional no qual estão envolvidas relações/interações/associações múltiplas e complexas entre os fatores individuais, sociais e relacionados ao sistema de saúde. Esses achados estão apresentados no modelo interpretativo elaborado no qual o diagrama representa o fenômeno "Enfrentando a complexidade do tratamento do Diabetes Mellitus e buscando o autocuidado" articulado com as dimensões do autocuidado das pessoas com DM2 e os aspectos facilitadores e dificultadores do autocuidado.

\section{CONCLUSÃO E IMPLICAÇÕES PARA A PRÁTICA}

A integração dos resultados dos estudos quantitativo e qualitativo propiciou a elaboração de um modelo interpretativo que ajuda na compreensão da adesão ao autocuidado de pessoas com DM2. O estudo quantitativo evidenciou baixas prevalências de adesão à dieta saudável, atividade física e à monitorização glicêmica, sendo estas explicadas na etapa qualitativa, que evidenciou muitas dificuldades nestas dimensões do autocuidado. As prevalências de adesão mais desejável ao uso da medicação e aos cuidados com os pés foram explicadas pela valorização dos aspectos facilitadores do tratamento nestas dimensões do autocuidado. $O$ estudo qualitativo mostrou que superar as dificuldades encontradas e valorizar os aspectos facilitadores do tratamento podem contribuir para que as pessoas com DM2 alcancem o autocuidado. Estes aspectos evidenciados nos estudos quantitativo e qualitativo são os elementos constituintes do modelo interpretativo elaborado.

Existe a compreensão de que os aspectos individuais, sociais e do sistema de saúde interferem no autocuidado. As dificuldades individuais e a vulnerabilidade social dessa parcela da população estudada fazem com que as pessoas com DM2 tenham uma forte dependência do sistema de saúde pública para os seus cuidados com a sua saúde. As dificuldades relacionadas à APS podem potencializar as dificuldades individuais e sociais, pois impedem o acesso a aspectos básicos do tratamento.

Quanto às limitações do estudo, ressalta-se que o fato de os resultados serem obtidos por autorrelato e referirem-se ao padrão de comportamento dos últimos sete dias, pelo instrumento utilizado, podem não representar a realidade referente à adesão ao autocuidado de pessoas com DM2.

A utilização do modelo interpretativo elaborado ajuda a compreender o fenômeno da adesão ao autocuidado das 
pessoas com DM2 atendidas na APS e podem contribuir, no contexto local, para o planejamento das ações de saúde destinadas a essa população. Dada a importância da APS na adesão ao autocuidado de pessoas com DM2, a relação entre os fatores estruturais e organizacionais do serviço e a adesão ao autocuidado entre as pessoas com DM2 precisa ser mais bem explorada por meio de outros estudos.

\section{CONTRIBUIÇÕES DOS AUTORES}

Desenho do estudo. Samara Eliane Rabelo Suplici. Betina Hörner Schlindwein Meirelles. Denise Maria Guerreiro Vieira da Silva. Julia Estela Willrich Boell.

Aquisição, análise de dados e interpretação dos resultados. Samara Eliane Rabelo Suplici. Betina Hörner Schlindwein Meirelles. Denise Maria Guerreiro Vieira da Silva. Julia Estela Willrich Boell.

Redação e revisão crítica do manuscrito. Samara Eliane Rabelo Suplici. Betina Hörner Schlindwein Meirelles. Denise Maria Guerreiro Vieira da Silva. Julia Estela Willrich Boell.

Aprovação da versão final do artigo. Samara Eliane Rabelo Suplici. Betina Hörner Schlindwein Meirelles. Denise Maria Guerreiro Vieira da Silva. Julia Estela Willrich Boell.

Responsabilidade por todos os aspectos do conteúdo e a integridade do artigo publicado. Samara Eliane Rabelo Suplici. Betina Hörner Schlindwein Meirelles. Denise Maria Guerreiro Vieira da Silva. Julia Estela Willrich Boell.

\section{EDITOR ASSOCIADO}

Beatriz Toso (1)

\section{EDITOR CIENTÍFICO}

Marcelle Miranda da Silva (1)

\section{REFERÊNCIAS}

1. Haas L, Maryniuk M, Beck J, Cox CE, Duker P, Edwards L et al. Nationa standards for Diabetes self-management education and support. Diabetes Care. 2013 jan;36(Supl.):100-8. http://dx.doi.org/10.2337/ dc13-S100.

2. American Diabetes Association. Introduction: standards of medical care in diabetes-2020. Diabetes Care. 2020 jan;43(Supl 1):S1-2. http:// dx.doi.org/10.2337/dc20-Sint.

3. Rincón-Romero MK, Torres-Contreras C, Corredor-Pardo KA. Adherencia terapéutica en personas con diabetes mellitus tipo 2. Rev. Cienc. Cuidad. 2017 jan;14(1):40-9. http://dx.doi.org/10.22463/17949831.806.

4. Albai A, Sima A, Papava I, Roman D, Andor B, Gafencu M. Association between coping mechanisms and adherence to diabetes-related self-care activities: a cross-sectional study. Patient Prefer Adherence. 2017;11:1235-41. http://dx.doi.org/10.2147/PPA.S140146.

5. Parra DI, Romero Guevara SL, Rojas LZ. Influential factors in adherence to the therapeutic regime in hypertension and diabetes. Invest Educ Enferm. 2019 out;37(3). http://dx.doi.org/10.17533/udea.iee.v37n3e02.

6. Salci MA, Meirelles BHS, Silva DMGV. Health education to prevent chronic diabetes mellitus complications in primary care. Esc Anna Nery. 2018;22(1):e20170262. http://dx.doi.org/10.1590/2177-9465ean-2017-0262.

7. Salci MA, Meirelles BHS, Silva DMGV. Primary care for diabetes mellitus patients from the perspective of the care model for chronic conditions. Rev latinoam. Enferm. 2017;25(0):e2882. http://dx.doi. org/10.1590/1518-8345.1474.2882
8. Santos CMJ, Faro A. Autoeficácia, lócus de controle e adesão ao tratamento em pacientes com diabetes tipo 2. Rev SBPH [Internet]. 2018; [citado 2020 out 11];21(1):74-91. Disponível em: http://pepsic.bvsalud org/scielo.php?script=sci_arttext\&pid=S1516-08582018000100005

9. Morin E. Introdução ao pensamento complexo. 4. ed. Porto Alegre: Sulina; 2011.

10. Creswell J. Projeto de pesquisa: método qualitativo, quantitativo e misto 3. ed. Porto Alegre: Art Med; 2010.

11. Charmaz K. A construção da teoria fundamentada: guia prático para análise qualitativa. Porto Alegre: Artmed; 2009.

12. Michels MJ, Coral MHC, Sakae TM, Damas TB, Furlanetto LM Questionário de Atividades de Autocuidado com o Diabetes: tradução, adaptação e avaliação das propriedades psicométricas. Arq Bras Endocrinol Metabol. 2010;54(7):644-51. http://dx.doi.org/10.1590/ S0004-27302010000700009.

13. Batista IB, Pascoal LM, Gontijo PVC, Brito PS, Sousa MA, Santos No $\mathrm{M}$ et al. Associação entre conhecimento e adesão às práticas de autocuidado com os pés realizadas por diabéticos. Rev Bras Enferm. 2020;73(5):e20190430. http://dx.doi.org/10.1590/0034-7167-20190430.

14. Saunders B, Sim J, Kingstone T, Baker S, Waterfield J, Bartlam B et al Saturation in qualitative research: exploring its conceptualization and operationalization. Qual Quant. 2018;52(4):1893-907. http://dx.doi. org/10.1007/s11135-017-0574-8.

15. Borba AKOT, da Silva MC, dos Santos SBS, Gomes MCR. Conhecimento e autocuidado de indivíduos com diabetes na Atenção Primária à Saúde. Rev. APS. 2018;21(4):516-33. http://dx.doi.org/10.34019/18098363.2018.v21.16072.

16. Boell JEW, Silva DMGV, Guanilo MEE, Hegadoren K, Meirelles BHS, Suplici SR. Resiliência e autocuidado em pessoas com diabetes mellitus. Texto Contexto Enferm. 2020;29:e20180105. http://dx.doi. org/10.1590/1980-265x-tce-2018-0105.

17. Souza JD, Baptista MHB, Gomides DS, Pace AE. Adesão ao cuidado em diabetes mellitus nos três níveis de atenção à saúde. Esc Anna Nery. 2017;21(4):e20170045. http://dx.doi.org/10.1590/2177-9465ean-2017-0045.

18. Machado APMC, Santos ACG, Carvalho KKA, Gondim MPL, Bastos NP, Rocha JVS et al. Avaliação da adesão ao tratamento de pacientes com diabetes mellitus e seus fatores associados. REAS. 2019;19(19, Supl. 1):e565. http://dx.doi.org/10.25248/reas.e565.2019.

19. Campos TSP, Silva DMGV, Romanoski PJ, Ferreira C, Rocha FL Fatores associados à adesão ao tratamento de pessoas com diabetes mellitus assistidos pela atenção primária de saúde. J Health Biol Sci. 2016;4(4):251. http://dx.doi.org/10.12662/2317-3076jhbs.v4i4.1030. p251-256.2016.

20. Casey M-B, Smart K, Segurado R, Hearty C, Gopal H, Lowry D et al Exercise combined with Acceptance and Commitment Therapy (ExACT) compared to a supervised exercise programme for adults with chronic pain: study protocol for a randomised controlled trial. Trials. 2018;19(1):194. http://dx.doi.org/10.1186/s13063-018-2543-5.

21. Sá GBAR, Dornelles GC, Cruz KG, Amorim RCA, Andrade SSCA, Oliveira T et al. O Programa Academia da Saúde como estratégia de promoção da saúde e modos de vida saudáveis: cenário nacional de implementação. Cienc. Saúde Colet. 2016 jun;21(6):1849-60. http:// dx.doi.org/10.1590/1413-81232015216.09562016.

22. Kolchraiber FC, De Souza Rocha J, Jovê César D, De Oliveira Monteiro $\mathrm{O}$, Andrade Frederico G, Antar Gamba M. Nível de atividade física em pessoas com diabetes mellitus tipo 2. Rev Cuid. 2018;9(2):2105-16. http://dx.doi.org/10.15649/cuidarte.v9i2.512.

23. Bermudez JAZ, Esher A, Osorio-de-Castro CGS, Vasconcelos DMM Chaves GCC, Oliveira MA et al. Assistência Farmacêutica nos 30 anos do SUS na perspectiva da integralidade. Cien Saude Colet. 2018;23(6):193749. http://dx.doi.org/10.1590/1413-81232018236.09022018.

24. Salin AB, Bandeira MSN, Freitas PRNDO, Serpa I. Diabetes Mellitus tipo 2: perfil populacional e fatores associados à adesão terapêutica em Unidades Básicas de Saúde em Porto Velho-RO. REAS. 2019 set;(33):e1257. http://dx.doi.org/10.25248/reas.e1257.2019.

25. Moreira SFC, Donato KDS, Silva LED, Lima KLL, Pelazza BB Borges $\mathrm{CJ}$ et al. Avaliação dos fatores relacionados à adesão de pacientes com Diabetes Mellitus ao tratamento. Rev. Itinerarius Reflectionis. 2018 dez;14(4):1-19. http://dx.doi.org/10.5216/rir. v14i4.54953.

26. Farias RFS, Lima AWS, Leite AFB, Santos ZCA, Santos ECB, Dias AA Adesão ao tratamento de Diabetes Mellitus em área rural do município de Vitória de Santo Antão - PE. Rev APS [Internet]. 2016 abr; [citado 2020 maio 11];19(2):181-90. Disponível em: https://periodicos.ufjf.br/ index.php/aps/article/view/15457 